\title{
Meeting Participants
}

(The number between parentheses is the guide to the meeting photograph.)

Adler, M. (4)

Jet Propulsion Laboratory

MS 301-180

4800 Oak Grove Drive,

Pasadena,

CA 91109-8099, USA

(madler@alumni.caltech.edu)

Adolfsson, L.G. (55)

Department of Astronomy,

University of Florida

211 SSRB,

Gainesville, FL 32611, USA

(adolfsso@astro.ufl.edu)

Anosova, J.

St. Peterburg, Russia

(anosova@clyde.as.utexas.edu)

Auer, S. (49)

A\&M Associates

1906 Paris Court,

Bowie, MD 20716, USA

Baggaley, J.W (23)

Dept. of Physics and

Astronomy,

University of Canterbury

Private Bag 4800,

Christchurch, New Zealand (phys051@csc.canterbury.ac.

$\mathrm{nz}$ )

Baguhl, M. (19)

Max Planck Institut für

Kernphysik

P.O. Box 103980,

69029 Heidelberg, Germany

(baguhl@dusty.mpi-hd.mpg.de)

Berriman, G.B. (37)

General Sciences Corporation

6100 Chevy Chase Dr.,

Suite 200,

Laurel, MD 20707, USA

(berriman@cobe1.gsfc.nasa.

gov)
Blum, J.

Max-Planck-Gesellschaft,

$A G$ "Staub in

Sternentstehungsgebieten",

University of Jena

Schillergaesschen 3 ,

D-07745, Jena, Germany

(blum@fred.astro.uni-jena.de)

Bradley, J. (56)

MVA Inc.

5500 Oakbrook Parkway,

Suite 200,

Norcross, GA 30093, USA

(jbrad@delphi.com)

Brown, $\mathrm{P}$ (5)

Department of Physics,

University of Western Ontario

London, Ontario N6A 3K7,

Canada

(peter@canlon.physics.uwo.ca)

Brownlee, D.E. (9)

Department of Astronomy,

University of Washington

Seattle WA 91195, USA

(brownlee@astro.washington.

edu)

Burns, J.A.

Cornell University

328 Space Sciences Building,

Itaca, NY 14853-6801, USA

(jab16@cornell.edu or

burns@astrosun.tn.cornell.edu)

Bussoletti, E. (38)

Istituto di Fisica Sperimentale,

Istituto Universitario Navale

Via A. De Gasperi 5,

80133 Napoli, Italy

(bussoletti@nava1.uninav.it)

Campins, $\mathrm{H}$.

Department of Astronomy,

University of Florida

211 SSRB,

Gainesville, FL 32611, USA

(campins@astro.ufl.edu)
Ceplecha, Z. (42)

Acad. of Sciences,

Astronomical Inst.,

Ondřejov Observatory

25165 Ondřejov, Czech Rep.

(ceplecha@asu.cas.cz)

Chatterjee, T. (83)

Facultad de Ciencias,

Fis.-Mat., Universidad

Autonoma de Puebla

Apartado Postal 1316,

72000 Puebla, Mexico

Clarke, D. (44)

University Observatory,

Dept. of Physics \& Astronomy

Acre Road, Glasgow,

G20 0TL, Scotland, UK

(dave@astro.gla.ac.uk)

Colangeli, L. (40)

Astronomical Observatory of

Capodimonte

Via Moiariello 16,

Napoli 80131, Italy

(colangeli@astrna.na.astro.it)

Cooper, B.L. (80)

Cooper \& Associates

1611 Dakota,

League City, TX 77573, USA

(cooper@sn3.jsc.nasa.gov)

Cruikshank, D.

NASA, Ames Research Center,

MS 245-6, Moffett Field, CA

94035-1000, USA

Dermott, S.F (86)

Department of Astronomy,

University of Florida

211 SSRB,

Gainesville, FL 32611, USA

(dermott@astro.ufl.edu) 
Dorschner, J. (29)

Max-Planck-Gesellschaft,

AG "Staub in

Sternentstehungsgebieten",

University of Jena

Schillergaesschen 3 ,

D-07745, Jena, Germany

(dorsch@georg.astro.uni-jena.

de)

Durda, D.D. (88)

Lunar \& Planetary Laboratory,

University of Arizona

Tucson, AZ 85721, USA

(durda@pirl.lpl.arizona.edu)

Dvorak, R.

Institut für Astronomie,

Universitätssternwarte

Tuerkenschanzstr. 17,

A1180 Wien, Austria

(dvorak@astro.ast.univie.ac.at)

Emel'yanenko, V V (34)

Armagh Observatory

College Hill, Armagh,

Northern Ireland, BT61 9DG

(ve@staru1.livjm.ac.uk)

Fajardo-Acosta, S.B. (79)

Division of Sicience,

Pennsylvania State University

Station Road, Erie,

Erie, PA 16563, USA

Farley, K.A. (64)

California Institute of

Technology

Pasadena, CA, USA

(farley@legs.gps.caltech.edu)

Finocchi, F (24)

Institut for Theoretical

Astrophysics,

University of Heidelberg

Neuenheimer Feld 561,

D-69120 Heidelberg, Germany

(frederic@ orion.ita.uni-heidel

berg.de)
Flynn, G.J. (45)

Physics Department,

Greenberg, J.M. (72)

State University of New York

at Plattsburg

Plattsburg, NY 12901, USA

Fomenkova, M. (76)

NASA Ames Research Center

MS 239-4, Moffett Field,

CA 94035-1000, USA

(marifo@cassir.ucsd.edu)

Garrett, $\mathrm{H}$.

Jet Propulsion Laboratory

MS 301-456

4800 Oak Grove Drive,

Pasadena,

CA 91109-8099, USA

Giovane, F. (48)

Code SZ, NASA Headquarters

Astrophysics Division,

300 E Street, SW,

Washington DC,

MD 20546-0001, USA

(fgiovane@helena.hq.nasa.gov)

Goidet-Devel, B. (71)

Observatoire de Besancon,

BP 1615, 25010 Besancon

Cedex, France

(blandine@obs-besancon.fr)

Gomes, R.S. (1)

Departamento de Astronomia,

Observatório Nacional

Rua General Jose Cristono 77, Hamilton, D.P. (16)

20941-400 Rio de Janeiro,

Brazil

(rodney@on.br)

Gor'kavyi, N.N. (2)

Crimean Astrophysical

Observatory

Simeiz, Yalta,

Crimea 334242, Ukraine

(gorkav@catpeak.crimea.ua)

Department of Astronomy

University of Maryland

College Park,

MD 20742-24.21, USA

(hamilton@astro.umd.edu)

Hanner, M.S. (70)

Jet Propulsion Laboratory

MS 183-601,

4800 Oak Grove Drive,

Pasadena,

CA 91109-8099, USA

(msh@scn1.jpl.nasa.gov) 
Harris, N.W (46)

Armagh Observatory

College Hill

Armagh, Northern Ireland,

BT61 9DG

(nwh@star.arm.ac.uk)

Hartl, $\mathrm{H}$.

Institut für Astronomie

University of Innsbruck

Technikerstrasse 25

A-6020 Innsbruck, Austria

Hauser, M.G.

NASA Code 680

Goddard Space Flight Center,

Greenbelt, MD 20771, USA

(Hauser@stars.gsfc.nasa.gov)

Hawkes, R.L. (78)

Physics Eng. \& Geology Dept.,

Mount Allison University

Sackville,

NB, E0A 3CO, Canada

(rhawkes@mta.ca)

Henning, Th. (47)

Max Planck Society,

$\mathrm{AG}$ "Staub in

Sternentstehungsgebieten",

University of Jena

Schillergaesschen 3,

D-07745, Jena, Germany

(henning@ fred.astro.uni-jena.

de)

Horn, L.J. (12)

Jet Propulsion Laboratory

MS 183-501

4800 Oak Grove Drive

Pasadena,

CA 91109-8099, USA

(lhorn@jpluvs.jpl.nasa.gov)

Hubener, W (41)

NASA Headquarters,

Solar System Division

300 E Street, SW,

Washington DC,

MD 20546-0001

(WALTER@swri.space.swri. edu)
Hughes, D.W (62)

Department of Physics,

Hicks Building,

University of Sheffield

Sheffield S3 7RH, UK

Ishimoto, H. (65)

Department of Earth and

Planetary Science,

Faculty of Sciences,

Kobe University

Nada, Kobe 657, Japan

(ishimoto@icluna.kobe-u.ac.jp)

Jayaraman, S. (87)

Department of Astronomy,

University of Florida

211 SSRB,

Gainesville, FL 32611, USA

(Jayaraman@astro.ufl.edu)

Jenniskens, P (26)

NASA Ames Research Center

MS 239-4,

Moffett Field,

CA 94035-1000, USA

(peter@max.arc.nasa.gov)

Jessberger, E.K. (6)

Max Planck Institut für

Kernphysik,

P.O. Box 103980

69029 Heidelberg, Germany

Kehoe, T. (22)

Astronomy Unit,

Queen Mary \& Westfield

College

Mile End Road

London E1 4NS, UK

Keller, H.U. (58)

Max Planck Institut für

Aeronomie,

Postfach 20, D-37189

Katlenburg-Lindau, Germany

(NSP::LINMPI::KELLER)

Keller, L.P (13)

MVA Inc.

5500 Oakbrook Parkway,

Suite 200,

Norcross, GA 30093, USA

(lpk111@delphi.com)
Kimura, H. (67)

Department of Earth and

Planetary Sciences,

Faculty of Science,

Kobe University

Nada, Kobe 657, Japan

(hiroshi@icluna.kobe-u.ac.jp)

Kitazawa, Y (32)

Machinery \& Structure

Development Dept.

Ishikawajima-Harima Heavy

Industries Co., Ltd.

1, Shin-Nakahara-Cho,

Isogo-Ku

Yokohama 235, Japan

(kitazawa@azalea.ihitri.ihi.co. jp)

Kortenkamp, S. (85)

Department of Astronomy,

University of Florida

211 SSRB,

Gainesville, FL 32611

(kortenkamp@astro.ufl.edu)

Kurat, G.

Naturhistorisches Museum

Postfach 417

A-1014 Vienna, Austria

Lamy, Ph.L. (33)

Laboratoire d'Astronomie

Spatiale, CNRS

BP8, 13376 Marseille,

Cedex 12, France

(lamy@astrsp-mrs.fr)

Levasseur-Regourd, A.-Ch.(84)

Service d'Aéronomie

BP3, 91371 Verrières le

Buisson, France

(chantal.levasseur@aerov.

jussieu.fr)

Liou, J.-C.

SN3, NASA Johnson Space

Center

Houston, TX 77058, USA

(liou@sn3.jsc.nasa.gov) 
Lynch, D.K. (51)

The Aerospace Corporation

P.O. Box 92957, M.S. M2/266

Los Angeles,

CA 90009-2957, USA

(lynch@qmail2.aero.org)

Maag, C.R.

T\&M Engineering

130 Ocean Garden Lane

Cape Canaveral,

FL 32920, USA

(cmaag@aol.com)

MacQueen, R.M.

Physics Department,

Rhodes College

2000 N. Parkway,

Memphis, TN 38112, USA

(rmac@rhodes.edu)

Mann, I. (14)

Max Planck Institut für

Aeronomie

Postfach 20, D-37189

Katlenburg-Linciau, Germany

(mann@linax 1.dnet.gwdg.de)

Masuda, K. (66)

Department of Earth and

Planetary Sciences,

Faculty of Science,

Kobe University

Nada, Kobe 657, Japan

(masuda@icluna.kobe-u.ac.jp)

Matney, M.J.

Lockheed Martin

Engineering and Sciences,

2400 NASA RD. 1

Mail Code C23

Houston, TX 77058-3799,

USA

Matson, D.L. (7)

Jet Propulsion Laboratory

MS 269-441,

4800 Oak Grove Drive

Pasadena, CA 91109, USA

(Dennis.L.Matson@jpl.nasa. gov)
Matthews, M. (68)

Dept. of Planetary Sciences,

Osip, D. (17)

Department of Astronomy,

Lunar \& Planetary Laboratory University of Florida

University of Arizona

211 SSRB,

Tucson, AZ 85721, USA

McDonnell, J.A.M. (15)

Unit for Space Sciences,

University of Kent

Canterbury.

Kent CT2 7NR, UK

(J.A.M.McDonnell@ukc.ac.uk)

Gainesville, FL 32611, USA

(osip@astro.ufl.edu)

Oliver, J. (77)

Department of Astronomy,

University of Florida

211 SSRB,

Mei, Y (61)

Jet Propulsion Laboratory,

MS 264-765,

4800 Oak Grove Drive,

Pasadena, CA 91109, USA

Messenger, S. (11)

Department of Physics,

Washington University

1 Brookings Drive,

St. Louis, MO 63130, USA

(scott@ionprobe.wash.edu)

Mukai, T (60)

Department of Earth and

Planetary Sciences,

Faculty of Science,

Kobe University

Nada, Kobe 657, Japan

(mukai@icluna.kobe-u.ac.jp)

Nakamura, R. (57)

Department of Earth and

Planetary Sciences,

Faculty of Science,

Kobe University

Nada, Kobe 657, Japan

(ryosuke@icluna.kobe-u.ac.jp)

Okamoto, H. (59)

Department of Earth and

Planetary Sciences,

Faculty of Science,

Kobe University

Nada, Kobe 657, Japan

(okamoto@komadori.earth.s.

kobe-u.ac.jp)
Gainesville, FL 32611, USA

(oliver@astro.ufl.edu)

Paul, K.G.

Fachgebiet Raumfahrttechnik,

TU-München

Richard-Wagner-Str. 18/III,

D-80333 München, Germany

(kpaul@asterix.lrt.mw.

tu-muenchen.de)

Pecina, P. (39)

Astronomical Institute of the

Czech Academy of Sciences

25165 Ondřejov. Czech Rep.

(ppecina@asu.cas.cz)

Pendleton, $Y$

Space Science Division,

NASA Ames Resech Center

Moffett Field,

CA 94035-1000, USA

(pendleton@gal.arc.nasa.gov)

Polansky C.

Jet Propulsion Laboratory

4800 Oak Grove Drive,

Pasadena,

CA 91109-8099, USA

(cpolanskey@gllsvc.jpl.nasa.

gov)

Reach, W.T. (36)

Laboratory for Astronomy and

Solar Physics, Code 680,

NASA Goddard Space Flight

Center

Greenbelt, MD 20771. USA

(reach@stars.gsfc.nasa.gov) 
Riemann, R. (20)

Max Planck Institut für

Kernphysik

P.O. Box 103980, 69029 Heidelberg, Germany

(riemann@kosmo.mpi-hd.mpg. de)

Sasaki, S. (73)

Geological Institute

University of Tokyo

Tokyo 113, Japan

(sho@geol.s.u-tokyo.ac.jp)

Sekanina, Z. (43)

Jet Propulsion Laboratory

4800 Oak Grove Drive,

Pasadena, CA 91109, USA

(zs@sek.jpl.nasa.gov)

Šimek, M. (28)

Astronomical Institute of the

Czech Academy of Sciences

25165 Ondřejov, Czech Rep.

(simek@asu.cas.cz)

Simon, C.

Precision Analytical Lab.

10218 SW 13th Pl,

Gainesville, FL 32607, USA

Srama, R. (18)

Max Planck Institut für

Kernphysik

P.O. Box 103980,

69029 Heidelberg, Germany

(srama@kosmo.mpi-hd.mpg.de

Steel, D.I. (30)

Department of Physics and

Mathematical Physics,

University of Adelaide

Adelaide, SA 5005, Australia

(dsteel@physics.adelaide.edu.

au)

Stognienko, R. (21)

Max-Planck-Gesellschaft,

AG "Staub in

Sternentstehungsgebieten",

University of Jena,

Schillergaesschen 3,

D-07745, Jena, Germany

(stog@fred.astro.uni-jena.de)
Svedhem, H. (82)

Space Science Department,

ESA/ESTEC, Postbus 299,

2200 Ag Noordwijk,

The Netherlands

(hsvedhem@estec.esa.nl)

Svestka, J. (25)

Prague Observatory

Petrin 205, 11846 Prague 1,

Czech Republic

Tanabe, H. (69)

1715-8 Takao-machi

Hachioji, Tokyo 193, Japan

Thomas-Osip, J.E. (91)

Department of Astronomy,

University of Florida

211 SSRB,

Gainesville, FL 32611, USA

(jet@astro.ufl.edu)

Toller, G. (52)

General Sciences Corp.

6100 Chevy Chase Dr.,

Laurel, MD 20707, USA

(toller@bikini.gsfc.nasa.gov)

Tsou, $\mathrm{P}$

Jet Propulsion Laboratory, 4800 Oak Grove Drive,

Pasadena, CA 91109, USA

(peter.tsou@jpl.nasa.gov)

Vaidya, D.B. (50)

Physical Research Laboratory,

Navrangpuram

Ahmedabad 380009

Gujarat, India

(dbvaidya@prl.ernet.in)

Vala, M.

Chemistry Department,

University of Florida

Gainesville, FL 32611, USA

Weissman, P (10)

Jet Propulsion Laboratory

MS 183-601,

4800 Oak Grove Drive,

Pasadena, CA 91109, USA

(pweissman@jpluvs.jpl.nasa.

gov)
Williams, I.P. (74)

Astronomy Unit,

Queen Mary \& Westfield

College

Mile End Road,

London E1 4NS, UK

(i.p.williams@qmw.ac.uk)

Worms, J.-C. (54)

ESSC-ENSPS

Parc d'Innovation,

Bd. Sebastien Brandt,

67400 Illkirch, France

(wormsjc@ensps.u-strasbg.fr)

Xing, $\mathrm{Z}$.

Jet Propulsion Laboratory

MS 183-501,

4800 Oak Grove Drive,

Pasadena, CA 91109

(xing@scn1.jpl.nasa.gov)

$\mathrm{Xu}$, Yin-Lin (75)

Purple Mountain Observatory

Space Research Div.,

2 W Beijing Rd.,

Nanjing, 210008 China

$\mathrm{Xu}, \mathrm{Yu}-\mathrm{lin}$ (89)

Department of Astronomy,

University of Florida

211 SSRB

Gainesville, FL 32611, USA

(shu@astro.ufl.edu)

Yamamoto, S. (27)

Department of Earth and

Planetary Sciences,

Faculty of Science,

Kobe University

Nada, Kobe 657, Japan

(yamachan@komadori.earth.s.

kobe-u.ac.jp)

Zook, H.A. (35)

SN3, NASA Johnson Space

Center,

Houston, TX 77058, USA

(zook@snmail.jsc.nasa.gov) 